\title{
Conceptual Uncertainty and Reasoning Tools
}

\author{
Bertil Rolf \\ Blekinge Institute of Technology, School of Management \\ SE-372 25 Ronneby, Sweden \\ bertil.rolf@bth.se
}

\begin{abstract}
Problems of conceptual uncertainty have been dealt with in theories of formal logic. Such theories try to accommodate vagueness in two main ways. One is fuzzy logic that introduces degrees of truth. The other way of accommodating formal logic to vagueness is super valuations and its descendants. This paper studies a more inclusive class of reasoning support than formal logic. In the present approach, conceptual uncertainty, including vagueness is represented as higher order uncertainty. A taxonomy of epistemic and conceptual uncertainty is provided. Finally, implications of conceptual uncertainty for reasoning support systems are analyzed.
\end{abstract}

\section{Introduction}

The purpose of computer based support systems for reasoning such as argumentation, decision and negotiation is to facilitate reasoning by replacing mental operations with externalized procedures, operating on representations in a computer medium. The design and development of computer support for reasoning, therefore, presupposes that we can find suitable external representations and describe procedures for operating on them.

A problem common to formal logic and more generally applicable reasoning procedures is the occurrence of conceptual uncertainty involving vagueness, fuzziness, ambiguity or open texture (Zadeh 1965, Fine 1975, Shapiro 2006). For instance, vagueness gives rise to problems of the law of excluded middle and of sorites paradoxes that are well known (Rolf 1981, Williamson and Graff 2002). If every proposition is true or false, is it true or false that France is hexagonal? If 0 grains do not make a heap but 100.000 grains do, which is the number $n$ such that $n$ grains did not make a heap but $n+1$ did? Such questions remind us that we are thinking by means of a conceptual system whose borders are not definitely sealed.

This paper studies the question: "How can computerized support systems for reasoning be accommodated to conceptual uncertainty? It asserts (1) Conceptual uncertainty is important for various types of reasoning such as argumentation, decision making and negotiations. (2) In reasoning supporting 
systems, conceptual uncertainty is representable as a second order uncertainty, directed towards the first order representations of matters of fact or courses of action. (3) There are five types or dimensions of uncertainty, two of them epistemic, three conceptual. The lens model of judgment theory confirms the taxonomy. (4) Conceptual and epistemic uncertainty can clarify reasoning in judgment and decision making. (5) Computer tools supporting reasoning need to consider both epistemic and conceptual uncertainty. This implies a need for a multiplicity of computer support tools together with corresponding skills to handle them.

\section{Conceptual Uncertainty in Reasoning and Decision Making}

In processes of reasoning and argumentation processes, there can occur two kinds of uncertainty (Smithson 1989, 2004). One is epistemic uncertainty consisting in uncertainty about what the facts are and what follows from what we know. Epistemic uncertainty is often represented in the form of a probability distribution with well-defined properties. Epistemic uncertainty is due to incompleteness of knowledge, i.e. ignorance.

Conceptual uncertainty is due to incompleteness of meaning, or intention. Even if you knew every single fact about a place - climate, vegetation or geology - you might still be uncertain whether it is "suitable for hiking". The latter uncertainty can only be reduced if we clarify whose hiking we are discussing and the preferences and abilities of those persons.

Conceptual uncertainty affects the two major models of rationality in decision making, consequentialist rationality and deontological rationality. Consequentialists think of decisions as a selection of alternatives. In such decisions, there will be conceptual uncertainty pertaining to alternatives and to attributes. Which alternatives are there and which attributes should one evaluate?

In deontological rationality, decisions are based on general principles such as laws, regulations, policies or instructions. Legal decision making is typical. In such decisions, conceptual uncertainty concerns which principles to apply and how to apply them, e.g. in case of conflicts between principles.

\section{Conceptual Uncertainty of Level $n$ Is Represented at $\mathbf{n}+1$}

Consider the type theory of Russell and Whitehead (Whitehead and Russell 1910). I will show how conceptual uncertainty relating to a factual assertion of the first level can be represented at the second level. Consider the assertion:

The solar system has nine planets. 
This statement was held true from 1930 when Pluto was discovered up till August 2006. Recently, however, Pluto has been found in peculiar company. The Kuiper belt, of which Pluto is a part, contains some 100000 other objects. Some of them have the size of Pluto and one is actually larger. Pluto is very different from the other eight planets of the solar system.

It is certain that Mercury, Venus, Earth, Mars, Jupiter, Saturn, Uranus, and Neptune are planets. But there has for some time been uncertainty in the community of astronomers whether the judgment:

Pluto is a planet.

is true or false. The uncertainty could stem from uncertainty about facts about Pluto or uncertainty about the conceptual delimitation of planethood. The uncertainty about the conceptual delimitation could not be resolved by knowledge about what the world is like.

In 2006, astronomers faced two possible delimitations of "planet”. One conceptual decision might be to define "planet" is a way that excludes Pluto and the rest of the objects in the Kuiper belt. Another conceptualization would include Pluto, but then it is problematic whether other objects of the Kuiper belt should be included as well.

The uncertainty about the planethood of Pluto could not be removed by a pure appeal to facts. It had to be removed in another way. The matter was settled by a decision at the XXVIth General Assembly of the International Astronomical Union (IAU) in August 2006 in Prague. The assembly had two options to define the concept of planet, one wider and one narrower. With a wider definition, based on a convention of 2005, the solar system would have contained twelve planets, including Pluto. But in 2006, the General Assembly of IAU settled for a narrower definition, stating that in the solar system, a planet is a celestial body that (1) is in orbit around the Sun, (2) has sufficient mass so that it assumes a hydrostatic equilibrium (nearly round) shape, and (3) has "cleared the neighborhood" around its orbit.

Together with the presently known facts, this definition settles that Pluto is not a planet. The solar system now contains eight planets, in the sense of "planet" defined in 2006.

The settling of the question whether Pluto is a planet is not of the same kind as the removal of other astronomical uncertainties or ignorance. Consider procedures for removing epistemic uncertainty about planets. In removing epistemic uncertainty, observations and inference from facts is used. The concept of "planet" is held fixed or, at least, not consciously elaborated upon. The planet Neptune was discovered in 1846 as a result of mathematical prediction. Perturbations in the orbit of Uranus led astronomers to deduce Neptune's existence. Applying analogous methods, the astronomer Urbain Le Verrier in 1859 tried to deduce a planet "Vulcan" that would have caused perturbations in the orbit of Mercury. There were several reports about observations of "Vulcan" up to 1915, when Einstein successfully explained the apparent anomaly in 
Mercury's orbit. As Einstein's explanation became accepted, the search for Vulcan was abandoned by astronomers.

Instead, in removing the conceptual uncertainty of planet in 2006, a conceptual decision was needed. This decision was not an arbitrary one. It would have implications for the whole system of astronomical knowledge about the solar system and its planets. One cannot decide to redefine the concept of planet wider so as to include Pluto without also getting extra, unwanted, planets. Furthermore, had the wider definition been taken, the solar system might have come to include 53 presently known bodies in the solar system and possibly some hundreds of similar objects, presently unknown (Pluto Wikipedia 2006).

In fact, the decision about the definition of "planet" has been contested. The U.S. state of New Mexico's House of Representatives passed a resolution declaring that Pluto will always be considered a planet while overhead of the state, with March 13th being known as "Pluto Planet Day". Local news report that the widow and daughter of Clyde Tombaugh, discoverer of Pluto in 1930, participated in a solemn ceremony to reinstate the planethood of Pluto.

The uncertainty about there being a planet "Vulcan" was an epistemic uncertainty. Knowledge about facts could eliminate the uncertainty, given the then prevailing sense of "planet". The uncertainty about Pluto that was ended or postponed by the IAU in Prague in August 2006 could not be ended by an appeal to facts. A conceptual decision either reinstating the previous sense of "planet" or settling a new sense was necessary to eliminate the conceptual uncertainty relating to the planethood of Pluto.

The uncertainty relating to the existence of a planet "Vulcan" is a first order uncertainty. The uncertainty concerns whether the facts are such as to make the following statement true:

There is a planet between Mercury and the Sun.

The uncertainty about the planethood of Pluto is a second order uncertainty. We can represent it as an uncertainty about which to choose of the following two ways of rendering the concept of planet.

1. The predicate "planet" expresses the properties: (A) is in orbit around the Sun, (B) has sufficient mass so that it assumes a hydrostatic equilibrium (nearly round) shape.

2. The predicate "planet" expresses the properties: (A) is in orbit around the Sun, (B) has sufficient mass so that it assumes a hydrostatic equilibrium (nearly round) shape, and (C) "cleared the neighborhood" around its orbit.

The uncertainty about the planethood of Pluto can be represented as an uncertainty between the choice of 1 or 2 as ways to fixate the content of the predicate "planet".

Let me sum up some of the features of elaborations of epistemic versus conceptual uncertainty. (1) In science, epistemic uncertainty is diminished or 
removed ultimately by observation or experiment concerning putative facts together with inference from such. (2) Consequently, when epistemic uncertainty is removed, one obtains first order knowledge about facts or the implications of such knowledge. (3) In science, conceptual uncertainty is removed by decisions about the conceptual or representational system. (4) In a rational enterprise, such as science, decisions settling conceptual uncertainty are not arbitrary. They are made with considerations about consequences for large parts of a representational system of knowledge. (5) Consequently, in science, elaborations of conceptual uncertainty involve metacognition, i.e. knowledge and procedures involving our own system of knowledge. Such metacognition involves procedures of the logical second order.

One can identify epistemic uncertainty about what we would need to establish in order to settle a matter holding the present conceptual system fixed. Or we can identify conceptual uncertainty about what we would need to establish in order to settle a matter relating to changes in our conceptual system, holding facts fixed. Epistemic and conceptual uncertainty are abstract dimensions - one might need to elaborate on both dimensions in a particular case. This distinction and the facts it relies on - seem to be in conflict with attempts to model vagueness as a kind of epistemic uncertainty. (cf Williamson 1994)

Similar considerations apply to normative systems such as law, regulations, policy, strategy or valuation. The two types of sources for uncertainty apply not only to cognition but also to values and norms. Administrative terminology draws on compromises between science, folk wisdom, supposedly moral and religious commands, legal considerations, and political expediency. Conceptual elaborations might be called for in order to treat proper things in a proper manner. An example is the legal concept of death. When medical technology enabled hospitals to maintain a person's bodily functions in spite of serious brain damage, legal decisions fixated the concept of death.

Can we generalize our analysis about conceptual uncertainty? Conceptual uncertainty relating to choice is also representable at the second order. A consequentialist decision maker can be uncertain about the set of alternatives, the set of attributes and the methods for selecting an alternative, e.g. by compensatory, trade off methods or by non-compensatory methods, e.g. by some lexicographic method. A deontologist decision maker can be uncertain about which principles to select for application. Thus, conceptual uncertainty for a consequentialist and for a deontologist can be represented as something that needs to be settled by second order considerations.

\section{A Taxonomy of Epistemic and Conceptual Uncertainty}

We have claimed that epistemic uncertainty is absence of factual knowledge, i.e. knowledge of the first order, that would settle a question such as there being a planet "Vulcan" between Mercury and the Sun. We have claimed that conceptual 
uncertainty is the absence of knowledge of higher order that would settle a question such as Pluto being a planet. Such knowledge would need to rely on a fixation of the meaning of the predicate "planet".

Can the distinction we introduced be generalized to a theory of a more general distinction between kinds of uncertainty? I claim it can. There are, it seems, the following dimensions involved in solving any problem:

A. What is the problem? Identify the target problem, i.e. find a formulation of a question that covers the desire to make up one's mind. The question can be factual, mathematical, volitional, or preferential.

B. On which factors does the problem depend? Identify the factors that potentially bear sufficient relevance to the target problem. This can be an unstructured list of factors that might contribute to settling the question expressing the target problem.

C. What is the form of dependency? Identify the general form of influence that these factors bear to the target problem. This can, for instance, be an equational system, decomposing the dependency of the target vector into a set of equations with parameters and variables.

D. What is the strength of dependency? Identify the direction and strength by which the factors contribute. If the representation is in the form of an equational system, this aspect involves settling the parameters occurring in the equations.

E. What are the facts on which the solution to the problem rests? Identify the values of the variables expressing the factors of dependence.

Of these five dimensions of uncertainty, the first three A-C involve uncertainty of higher order and D and $\mathrm{E}$ involve uncertainty about facts, i.e. uncertainty of first order.

\section{Arguments for the Taxonomy}

Why these five and not three or seven dimensions of uncertainty? It is connected to the way that modeling can provide answers to problems. One needs to articulate the problem (A), the factors (B) and dependencies (C) that may be of importance. Finally, facts about the world and about preferences or norms of decision makers will settle the question, i.e. (D) and (E). The dimensions A-E are what it takes to settle a problem (cf. Nickles 1981).

The taxonomy clarifies the distinction between well structured problems and ill structured problems. Well structured problems are problems where A-C are settled while $\mathrm{D}$ and $\mathrm{E}$ need to be settled and there are known methods for settling D and E (Buckingham Shum 2003). Ill structured problems are open in 
some of the respects A, B or C. Those problems cannot be solved merely by an appeal to facts like $\mathrm{D}$ and $\mathrm{E}$ can.

The taxonomy fits the lens model of judgment theory. The model studies a subject utilizing certain cues to pass judgment on an object or a state. The cues can bear more or less ecological validity as indicators of the object/state. The subject may utilize these cues better or worse to achieve accuracy in her judgment. The accuracy can be represented as the product of ecological validity and cue utilization. The lens model has been applied to several types of judgment, from perceptual judgments via prognoses and predictions about the future (Payne 1993, Cooksey 1996, Hastie and Dawes 2001, Stewart 2000). The lens model can be used both descriptively and normatively. The cue utilization describes which weights a human judge or group of such lay to certain cues or indicators. If those weights are assigned in accordance with the ecological validity, one can achieve maximal accuracy in one's judgment, i.e. maximal rate of agreement with the real object or state about which one passes judgment.

The lens model represents a decomposition of our knowledge about facts and relations. The model can be expressed in two equations. One equation represents the ecological validity of the cues, i.e. how well knowledge of the cues would enable a judge to pass veridical judgment on the state of the object. The other equation represents the actual utilization of those cues by a human judge (or group of such).

Interestingly, these two equations can normally be represented by two linear functions. The cues that indicate the state of the object can be measured on scales that often permit a regression analysis, resulting in a linear function. Often, such a linear function represents the maximal accuracy that any human judge can reach (Dawes and Corrigan 1974).

A specific lens model, tied to an expert or group of experts forming judgment can be realized via the five dimensions of fixation. Any lens model will form a set of equations. Therefore, the five dimensions automatically apply.

\section{Applications of the Taxonomy}

Below, I will show the applicability of the taxonomy of conceptual uncertainty. A first way in which conceptual uncertainty enters into decision making is via the articulation of the target problem. For instance, one may ask whether the target factor can be spatially, temporally or causally subdivided. In an evaluation of Swedish measures taken against eutrophication in the Baltic, an international expert committee divided the target area into: (1) The Swedish east coast, (2) The open Baltic proper, (3) The Swedish west coast, (4) The Bothnian Bay, and (5) The Bothnian Sea (Eutrophication of Swedish seas 2006).

The division made is spatially and causally based. The Bothnian Bay and the Bothnian Sea do not seem to be affected of eutrophication. The mechanisms producing eutrophication and its consequences are different at the Swedish west 
coast were salt water prevents cyanobacterial blooms. A mixed set of measures is recommended and differences of causal mechanisms make different measures applicable at different places.

Sometimes, the articulation of the target problem can be facilitated via an articulation of the underlying qualities of ideal and of worst-case alternatives (Hammond et al. 1999). By so doing, one can come to an understanding of the dimensions underlying the decision at hand. Consider, for instance, eutrophication of the Baltic, a development everyone considers undesirable. But what is there about it that makes it undesirable: the deterioration of incomes from fishing, the massive algal blooms in the coastal regions or massive loss of oxygen-dependent life in the Baltic? By spelling out such underlying dimensions of the problem, one can, possibly, discover various sets of measures, relevant in different time spans.

A second way in which conceptual uncertainty enters is via the factors relevant to the target problem. One way to bring out factors of relevance uses top down procedures starting with definitions and other ways of making complex factors explicit. For instance, eutrophication is a concept that can be defined in a number of ways:

Eutrophication, however, is a condition in an aquatic ecosystem where high nutrient concentrations stimulate the growth of algae, which leads to imbalanced functioning of the system, such as: (1) intense algal growth: excess of filamentous algae and phytoplankton blooms; (2) production of excess organic matter; (3) increase in oxygen consumption; (4) oxygen depletion with recurrent internal loading of nutrients; and (5) death of benthic organisms, including fish (HELCOM Stakeholder Conference on the Baltic Sea Action Plan 2006).

Eutrophication is a process whereby water bodies, such as lakes, estuaries, or slow-moving streams receive excess nutrients that stimulate excessive plant growth (algae, periphyton attached algae, and nuisance plants weeds). This enhanced plant growth, often called an algal bloom, reduces dissolved oxygen in the water when dead plant material decomposes and can cause other organisms to die (Eutrophication US Geological Survey 2006).

Difference in wording may direct focus towards different aspects and point towards various solutions to a problem. We can compare the two underlying definitions: 


\begin{tabular}{|l|l|l|}
\hline & \multicolumn{1}{|c|}{ HELCOM } & \multicolumn{1}{|c|}{ U.S.G.S. } \\
\hline $\begin{array}{l}\text { What kind of things can } \\
\text { undergo eutrophication? }\end{array}$ & an aquatic ecosystem & water bodies \\
\hline $\begin{array}{l}\text { What is the ontology of } \\
\text { eutrophication? }\end{array}$ & $\begin{array}{l}\text { a condition with } \\
\text { certain consequences }\end{array}$ & $\begin{array}{l}\text { process of delivery } \\
\text { plus growth }\end{array}$ \\
\hline What characterizes the entity? & high concentrations & excess nutrients \\
\hline
\end{tabular}

Fig. 1. Comparison between two definitions of Eutrophication, HELCOM vs. U.S.G.S.

Eutrophication is in HELCOM something that befalls aquatic ecosystems and such systems are not strictly identifiable with water bodies as U.S.G.S does. An ecosystem is causally delimited while a water body is spatially and temporally delimited. Concentrations of nutrients as high or in excess both involve comparisons but perhaps with different units. A concentration can be high without being in excess and conversely. While HELCOM focuses on a condition with its effects, U.S.G.S. focuses on a causal process of nutrient delivery and growth effect.

The slightly different definitions may open or close different possible activities to counter eutrophication. From the definitions, it would seem that HELCOM is committed to countermeasures for bringing concentrations down and preserving ecosystems, while U.S.G.S. might operate by introducing ecosystems consuming nutrients.

A third way in which conceptual uncertainty enters into decision making is via the general structure of dependence. As pointed out by Herbert Simon (Simon 1996, Chap. 8), our analysis and insight into complex systems rests on the assumption that they are nearly hierarchically decomposable. Most of his claims can be read as an epistemic or perhaps pragmatic claim about useful simplifications in causal system modeling (Agre 2003). For instance, in one example, he considers causal decomposability of the eutrophication process of Lake Erie. One way of representing eutrophication would be to build a complex simulation of interaction between grids in the lake, using equations to represent phosphate production and phosphate usage in each cell of the grid. Massive amounts of data about sewage plants and river mounds would be needed. Furthermore, predictions about growth of urban population, industry and agriculture would be needed in such a conception of modeling.

Instead, Simon suggests a temporal decomposition into three kinds of sub processes, one of long term change, one of short term change and one of intermediate term change. The long-term change is, Simon claims, gradual and hardly noticeable during human life spans. It is not needed in the model. The fastest process is the eutrophication. Therefore, Simon suggests that modeling should disregard other dynamic aspects underlying the whole process that are ill understood. The resulting, simplified model focuses on the relation between 
phosphate input and eutrophication. Finally, he notes that phosphate levels in the parts of the lake are strongly intercorrelated, so models can disregard differences between grids. Furthermore, human activities related to sewage treatment are too gross to be finely modeled. Simon concludes that more finely granulated models are superfluous to "social purposes” (Simon 1997 p. 109-111).

In Simon's example of a modeling process, decomposition serves the purpose of simplification of prediction with reasonable precision. "Our concern is not to forecast the future but (a) to understand the consequences of alternative possible futures, and (b) to understand which of these possible futures is associated with particular strategies or policy measures" (ib. p. 112). More interesting than prediction is sensitivity and an understanding of which the key variables are and what policy variables can have an effect, Simon holds.

As Simon indicates, the dependencies could be rendered in a modeling process with far more detail and with the ambition to predict. The general structure of dependence that Simon suggests has low granularity. Simon defends the lack of detail. We are ignorant about facts and causal relations, which make more fine-grained predictions impossible. Hence, first order uncertainty about facts interacts with second order uncertainties and second order decisions about the granularity of the representing system.

\section{Computerized Tools for Judgment and Decisions}

Do the concepts epistemic and conceptual uncertainty with our taxonomy help develop reasoning skills and design support tools? If so, in what way?

First, a complete computer support for reasoning skills must enable the representation and elaboration not only of epistemic uncertainty but also of conceptual uncertainty. Typically, decision support systems deal with the reduction of epistemic uncertainty only. Procedures for elaborating on epistemic support do not, by themselves, contribute to elaboration of conceptual uncertainty. But conceptual uncertainty is pervasive in decision making processes. We have already mentioned the conceptualization and selection of alternatives and attributes necessary in decision making.

Second, conceptual uncertainty is of a higher order than an uncertainty about facts. The representation of such uncertainty can, in many cases, be performed via multiple representations, e.g. a multitude of decision trees or of a multitude of delimitations of an ambiguously described factor or a vaguely indicated relation. This feature of uncertainty is compatible with super valuation approaches that represent vagueness or ambiguity as sets of precise models. It is doubtful whether these considerations are compatible with fuzzy logic, fuzzy set theory or other one-dimensional ways of representing vagueness. An uncertainty concerning the definitions of "planet" fits super valuation approaches but not a graded approach between two extremes, typical of fuzzy logic approaches. 
Third, reasoning can involve rotations between procedures for elaborations on epistemic uncertainty and procedures for elaborating on conceptual uncertainty. For instance, a district attorney can be in doubt whether to press a charge of murder, manslaughter or severe assault. The burden of proof for the respective charges will differ. There is conceptual uncertainty about how to describe the deed together with epistemic uncertainty about what the facts of the case really are. Interaction between kinds of uncertainty obstructs sequential procedures for such rotation (But cf. Thagard 1992).

Fourth, in reasoning with conceptual uncertainty, one needs to be able to elaborate on multiple representations simultaneously. A problem can be symbolized in various ways and a decision maker can be uncertain about the best ways to construct or select a representation. Especially, multiple parties in a reasoning process can opt for different representations that, nevertheless, make competing claims about being the best ways to represent the (unarticulated) problem at hand. Therefore, a computer based reasoning supporting system should allow comparison between various representations (Rolf 2006a, 2006b).

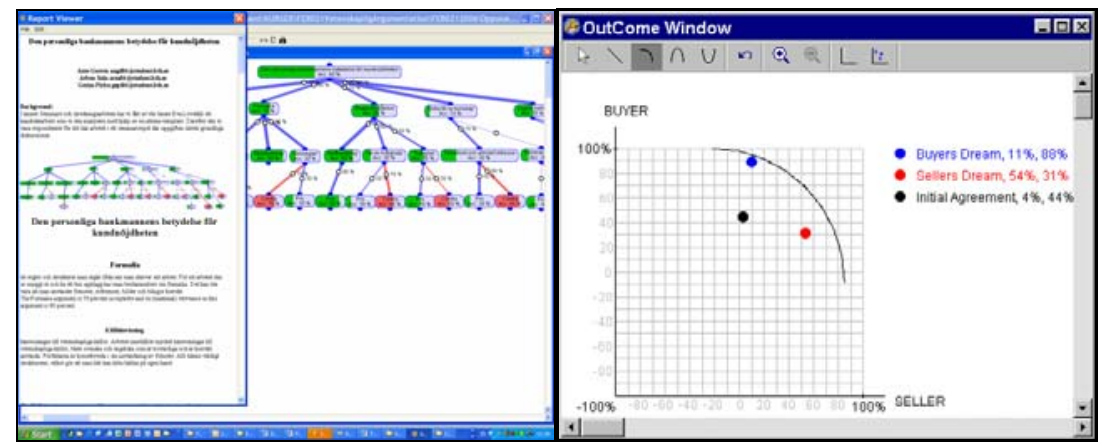

Fig. 2. Athena Standard (left), showing tree graph with report viewer presenting text output. Athena Negotiator (right), showing outcome diagram for two-party negotiation (Rolf 2002)

Human reasoning needs to be supported by multiple tools, providing multiple representations in order to represent conceptual uncertainty.

Finally, a computer-based support system will function as a tool in many respects. Each tool is part of a toolbox. There is no universal tool for all kinds of reasoning support. The various subtypes of uncertainty may have to be elaborated upon with different tools. The use of each tool needs competence, i.e. procedural knowledge. The use of the whole toolbox involves an overview of the purposes to which each tool can be put. When a designer introduces a software package for reasoning support, therefore, it is desirable to bear in mind that someone needs to supply heuristics for applying each of the tools as well as the whole toolbox. 


\section{References}

Agre, P.E.: Architecture of complexity. Journal of the Learning Sciences Vol. 12 No. 3 (2003) 413- 426

Buckingham Shum, S.: The roots of computer supported argument visualization. In: Visualizing argumentation: software tools for collaborative and educational sensemaking (Kirschner, P. et al. (eds.)). Springer, London, 2003

Cooksey, R.W.: Judgment analysis. Academic Press, San Diego, CA, 1996

Dawes R., Corrigan, B.: Linear models in decision making. Psychological Bulletin 812 (1974) 95-106

Eutrophication. U.S. Geological Survey, U.S.G.S. http://toxics.usgs.gov/definitions/eutrophication.html (2006)

Eutrophication of Swedish seas. Report 5509. The Swedish Environmental Protection Agency, Stockholm, 2006.

Fine, K.: Vagueness, truth and logic, Synthese 30 (1975) 265-300

Graff, D., Williamson, T.: Vagueness. Ashgate-Dartmouth, Hants, UK, 2002

Hammond, J.S. et al.: Smart choices. Harvard Business School Press, 1999

Hastie, R., Dawes, R.M.: Rational choice in an uncertain world. Sage Publications, Thousand Oaks, CA, 2001

HELCOM. Stakeholder Conference on the Baltic Sea Action Plan, Helsinki, Finland, 2006

Nickles, T.: What is a problem that we may solve it? Synthese 47 (1981) 85-118

Payne, W et al.: The adaptive decision maker. Cambridge Univ. Press, Cambridge, 1993

Rolf, B.: Topics on vagueness. Studentlitteratur, Lund, Sweden, 1981

Rolf, B.: Athena software. http://www.athenasoft.org (2002)

Rolf, B.: Decision support tools and two types of uncertainty reduction. In: Effective environmental assessment tools (Emmelin, L. (ed.)). Blekinge Tekniska Högskola, Karlskrona, Sweden, Report 1 (2006a) 134-157

Rolf, B.: Logic software for apprenticeship in rough reasoning. In: Proceedings SICTTL. Univ. of Salamanca, Salamanca, Spain, 2006b.

Shapiro, S.: Vagueness in context. Clarendon Press, Oxford, 2006

Simon, H.A.: The sciences of the artificial. $3^{\text {rd }}$ ed. The MIT Press, Cambridge MA, 1996

Simon, H.A.: Models of bounded rationality. Vol. 3. The MIT Press, Cambridge, MA, 1997

Smithson, M.: Ignorance and uncertainty. Springer, New York, 1989

Smithson, M.: Ignorance and uncertainty. In: Causality, uncertainty \& ignorance. $3^{\text {rd }}$ International Summer School, University of Konstanz, Germany. http://www.unikonstanz.de/ppm/summerschool2004/program.htm\#smithson (2004)

Stewart, T.R.: Uncertainty, judgment, and error in prediction. In: Prediction: science, decision making, and the future of nature (Sarewitz, D. et al. (eds.)). Island Press, Washington DC, 2000

Thagard, P.: Conceptual revolutions. Princeton University Press, Princeton, NJ, 1992

Whitehead, N. A., Russell, B.: Principia mathematica. Cambridge Univ. Press, London, 1910

Wikipedia.: Pluto. www.wikipedia.org (2006)

Williamson, T. (1994): Vagueness, Routledge, London.

Zadeh, L.: Fuzzy sets. Information and Control 8, (1965) 338-353

Acknowledgments. This work has been granted support from the Swedish Environmental Protection Agency. 